\title{
Calidad de las espirometrías en un estudio epidemiológico de terreno. Factores determinantes de la necesidad de repetir el examen en el estudio Platino-Chile
}

\author{
MARCELA ARAYA B.*, JULIO PERTUZÉ R.**, GONZALO VALDIVIA C.*, \\ ANA MARÍA BATISTA M.***, ROGELIO PÉREZ P.****, HILDA FUENTES Y.*, ALEJANDRO JARA V.*, \\ MARÍA N. MÁRQUEZ*****, MÓNICA CIFUENTES S.* y CARMEN LISBOA B.**
}

\section{SPIROMETRIES IN A POPULATION BASED STUDY: FACTORS DETERMINING THE NEED OF TEST REPETITION, IN CHILE'S PLATINO STUDY}

Spirometry, the most used test to evaluate pulmonary function, is only occasionally measured in field epidemiological studies. Our aim was to determine which of the following factors in the Platino study can be associated low quality spirometries in the first session: sex, age, socioeconomic level, educational level, body mass index, cold chill, smoking, operating technician, date and subject's previous spirometric experience. 1.168 individuals were evaluated with spirometry. Fifteen nurses and 1 midwife were trained according to NIOSCH standards. Easy One NDD spirometers were used. 1.037 subjects (88.8\%) performed a satisfactory spirometry in the first session, and 131 (11.2\%) failed to do so. Only two significant predictors of this failure were identified: age and educational level. Causes for test repetition were 1) Non satisfactory acceptability (77.1\%); 2) lack of reproducibility (67.9\%); 3) decline of post bronchodilator CVF without concomitant change in $V E F_{1}(36.6 \%)$. Eighty nine of the 131 subjects accepted to repeat the test. A satisfactory spirometry was obtained in 79 subjects. Hence, at the end of the study $95.5 \%$ of the subjects attained a satisfactory test. We conclude that personnel without experience, with appropriate training, can perform high quality field spirometries. Acceptability and repoducibility were the most sensitive factors associated with a bad performance of spirometry. Age and schooling were the main factors related with a low quality spirometry. No association was detected regarding technician as a predictor of low quality spirometries carried out in a population setting.

Key words: Epidemiological field study, COPD, spirometry, quality control

\section{RESUMEN}

La espirometría es el examen más utilizado para evaluar la función pulmonar y ocasionalmente se usa en estudios epidemiológicos. Evaluamos si las variables: sexo, edad, nivel socioeconómico, escolaridad, IMC, cursar concomitantemente con

\footnotetext{
* Departamento de Salud Pública Pontificia Universidad Católica de Chile.

** Departamento de Enfermedades Respiratorias, Pontificia Universidad Católica de Chile.

*** $\quad$ Universidad Federal de Pelotas, Brasil. Coordinadora proyecto Platino.

**** Instituto Nacional de Enfermedades Respiratorias de México, México DF.

***** Escuela Universitaria de Tecnología Médica. Facultad de Medicina. Hospital de Clínicas, Montevideo, Uruguay.
}

Este trabajo es parte del estudio multicéntrico de EPOC en 5 grandes ciudades de América Latina (THE PLATINO STUDY), coordinado por la Asociación Latinoamericana del Tórax y apoyada por Boheringer Ingelheim. 
resfrío, tabaquismo, fecha del examen, haberse efectuado espirometría anteriormente y técnico responsable, se asociaban con la probabilidad de espirometrías insatisfactorias en una primera sesión. Quince enfermeras y una matrona que recibieron capacitación realizaron las espirometrías en 1.168 sujetos empleando un espirómetro Easy One NDD. En la primera sesión 1.037 individuos $(88,8 \%)$ efectuaron una espirometría satisfactoria fracasando 131 (11,2\%). Se identificaron dos variables predictoras del fracaso: edad y años de instrucción. Las causas más frecuentes de espirometrías insatisfactorias fueron: 1) No cumplir con criterios de aceptabilidad (77,1 \%); 2) Falta de reproducibilidad (67,9\%); 3) Caída de CVF post broncodilatador sin cambio en el $V E F_{1}(36,6 \%)$. De los 131 sujetos cuya espirometría fue insatisfactoria en la primera sesión, ochenta y nueve aceptaron repetirla, lográndose al final del estudio una espirometría satisfactoria en el 95,5\% de la muestra. Personal técnico sin experiencia previa y adecuadamente entrenado, es capaz de lograr espirometrías de buena calidad en un estudio epidemiológico de campo. Las causas más frecuentes de repetición de examen guardan relación con dificultad de alcanzar criterios de aceptabilidad y reproducibilidad de la prueba. Factores gravitantes en la repetición son la edad y el nivel de instrucción de los sujetos examinados. El desempeño de los técnicos no constituyó un factor limitante para obtener una adecuada calidad de la espirometría en terreno.

Palabras clave: Estudio epidemiológico de campo; EPOC; espirometría; control de calidad.

\section{INTRODUCCIÓN}

La espirometría es el examen más utilizado para evaluar la función pulmonar la cual se realiza mayoritariamente en laboratorios especializados y ocasionalmente en estudios de investigación poblacional.

La calidad técnica de la espirometría es muy relevante por cuanto una prueba insatisfactoria podría inducir a errores de interpretación tanto en el diagnóstico como en el tratamiento ${ }^{1}$. Es así como la variabilidad observada entre mediciones efectuadas por diferentes operadores puede superar la diferencia esperada por efecto de una intervención terapéutica. Por otra parte, un alto nivel de imprecisión y variabilidad en las mediciones pueden ocasionar errores importantes cuando se estudian factores etiológicos cuyo efecto es cuantitativamente pequeño, pero de relevancia clínica o epidemiológica ${ }^{2,3}$.

Los factores que determinan el mejor desempeño de un técnico no son bien conocidos, pero sin duda el entrenamiento adecuado es un factor que contribuye a mejorar su rendimiento. Se ha demostrado que la variabilidad en el desempeño de los operadores tiende a disminuir a medida que avanza el desarrollo de un protocolo de investigación ${ }^{4}$.

Considerando la escasez de estudios poblacionales que incluyan espirometrías entre sus mediciones, nos pareció de interés evaluar la influencia de algunas variables que puedan determinar la calidad de este examen y la necesi- dad de repetirlo, en el Proyecto Latinoamericano de Investigación en Obstrucción Bronquial (PLATINO), investigación de terreno destinada a establecer la prevalencia de enfermedad pulmonar obstructiva crónica (EPOC) en la ciudad de Santiago.

El propósito de esta comunicación fue evaluar los factores que inciden en la obtención de resultados confiables en términos de aceptabilidad y reproducibilidad ${ }^{5,6}$ en espirometrías efectuadas en terreno por personal recientemente entrenado.

\section{SUJETOS Y MÉTODOS}

El proyecto PLATINO es un estudio multicéntrico destinado a evaluar la prevalencia de EPOC en población adulta de 40 y más años residente en hogares de áreas urbanas de grandes ciudades de América Latina (Sao Paulo, Ciudad de México, Montevideo, Santiago de Chile y Caracas). Su centro coordinador se sitúa en Brasil y utiliza un protocolo consensuado, sujeto a un estricto control de calidad in situ. Los detalles metodológicos han sido recientemente comunicados ${ }^{7}$.

En cada centro se utilizó un muestreo aleatorio multietápico, por conglomerado y estratificado según nivel socioeconómico. El Instituto Nacional de Estadísticas de Chile seleccionó un total de 1.019 viviendas correspondientes a 34 comunas del gran Santiago. A cada participante se 
le aplicó un cuestionario estructurado a partir de instrumentos previamente validados ${ }^{8-11}$ para evaluar su estado general de salud. Los instrumentos en español fueron validados tanto en el centro coordinador como localmente en Santiago.

A partir del cuestionario se estudió la presencia de algunas variables que pudieran guardar relación con la capacidad de realizar una espirometría aceptable y reproducible tales como sexo, edad, nivel de escolaridad, tabaquismo activo y nivel socioeconómico. Además, se consideró variables antropométricas obtenidas a través de mediciones estandarizadas de peso y talla, calculándose el índice de masa corporal (IMC).

Para las determinaciones se emplearon 19 espirómetros portátiles NDD que cuentan con un microprocesador EasyOne ${ }^{\mathrm{TM}}$ (Medical Technologies, Chelmsford, Massachusetts and Zürich, Switzerland) cuyas mediciones de volumen y flujo se basan en la determinación del tiempo de tránsito de partículas gaseosas por método ultrasónico ${ }^{12}$. La exactitud de cada espirómetro fue revisada diariamente utilizando una jeringa de tres litros, con un margen aceptable de error de $1,6 \%$ (con un rango entre 2.950 y $3.050 \mathrm{ml}$ ).

La entrevista inicial comprendió la aplicación de un breve cuestionario para determinar si el sujeto era elegible para realizar la espirometría. Se consideró criterio de exclusión espirométrico el embarazo, antecedentes en los últimos tres meses de: cirugía torácica o abdominal, infarto del miocardio, desprendimiento de retina, hospitalización por causa cardiovascular, personas en tratamiento por tuberculosis y sujetos con frecuencia cardiaca sobre 120 por minuto.

Los participantes elegibles realizaron un máximo de 8 maniobras de inspiración máxima seguida de una espiración forzada hasta obtener tres maniobras aceptables con una máxima variación de $150 \mathrm{ml}$ en la capacidad vital forzada (CVF) y en el volumen espiratorio forzado primer segundo $\left(\mathrm{VEF}_{1}\right)^{2}$. Posteriormente, se administró $200 \mu \mathrm{g}$ de salbutamol, por vía inhalatoria, a través de una aerocámara de $320 \mathrm{ml}$ de capacidad, repitiéndose la espirometría 15 minutos después. La espirometría se realizó en posición sentada, con el individuo respirando a través de una pieza bucal desechable y con la nariz ocluida por una pinza nasal.

\section{Entrenamiento del personal responsable de la actividad de terreno}

Quince enfermeras y una matrona, sin experiencia previa en espirometrías, recibieron durante un período de 3 días información sobre los aspectos teóricos y prácticos del proyecto a realizar, nociones básicas de fisiología respiratoria y capacitación tanto en ejecución de espirometrías como en mediciones antropométricas. En una etapa inmediatamente posterior, se efectuó el entrenamiento definitivo de una semana de duración que incluyó 2 días de entrenamiento formal en espirometría realizado por dos expertos del NIOSH (National Institute for Occupational Safety and Health, USA) provenientes de México. Las 16 profesionales responsables de la actividad en terreno recibieron la certificación correspondiente tras rendir un examen práctico. Previo al examen realizaron un mínimo de 10 espirometrías satisfactorias, tanto en sujetos normales como en pacientes, supervisadas directamente por los expertos.

Para la certificación antropométrica se estableció como niveles aceptables de variación intra e inter observador $0,2 \mathrm{~cm}$ en la talla y $200 \mathrm{~g}$ en el peso ${ }^{13-15}$.

El control de calidad de las espirometrías en terreno consideró los siguientes criterios:

a) Reproducibilidad de la maniobra: menos de $150 \mathrm{ml}$ de diferencia entre los dos mejores valores de $\mathrm{VEF}_{1}$ y $\mathrm{CVF}^{2}$

b) Aceptabilidad: obtención de 3 curvas aceptables según criterios de la $\mathrm{ATS}^{5}$. Para la evaluación de aceptabilidad se considera a su vez los siguientes criterios:

1) un volumen extrapolado inferior a $150 \mathrm{ml}$ o menor a $5 \%$ de la CVF;

2) diferencias en el flujo espiratorio máximo $(\mathrm{PEF})<10 \%$;

3) tiempo espiratorio mayor a 6 segundos;

4) curvas sin esfuerzo variable;

5) ausencia de tos durante la maniobra.

c) Ausencia de caída de la CVF post broncodilatador sin una disminución concomitante del $\mathrm{VEF}_{1}$, situación que puede determinar una relación $\mathrm{VEF}_{1} / \mathrm{CVF}$ post broncodilatador superior a 0,70 que no estaba presente en la espirometría basal. (Falsa respuesta broncodilatadora derivada de una menor $\mathrm{CVF}$ post $\mathrm{BD}$ sin cambio en el $\left.\mathrm{VEF}_{1}\right)$.

\section{Actividad en terreno}

Las evaluaciones en terreno fueron efectuadas por enfermeras en el domicilio de las personas seleccionadas siendo supervisadas aleatoriamente por alguna de las tres enfermeras coordinadoras del trabajo en terreno. Los equipos se calibraron diariamente en la mañana y la información de la espirometría se traspasó cada día a la base de datos. La evaluación técnica de la calidad de la espirometría estuvo a cargo de tres médicos especialistas durante todo el protocolo, 
quienes analizaron cada examen utilizando los mismos criterios, definiendo la necesidad de repetirlo, si éste no satisfacía los requerimientos técnicos descritos anteriormente. El protocolo fue aprobado por el comité de ética de la Comisión de Investigación de la Facultad de Medicina de la Pontificia Universidad Católica y cada participante firmó un consentimiento informado.

\section{Análisis estadístico}

La necesidad de repetir la espirometría posterior a un primer intento se analizó como variable dependiente y las variables explicatorias relacionadas con el sujeto estudiado fueron: edad, sexo, índice de masa corporal $\left(\mathrm{kg} / \mathrm{m}^{2}\right)$, años de instrucción, nivel socioeconómico (alto, medio, bajo), presencia de infección respiratoria aguda, tabaquismo actual y antecedente de realización previa de espirometría. Como variable derivada del técnico responsable de la espirometría se analizó el número de espirometrías que tuvo que repetir cada técnico y como variable explicativa el número de días transcurrido desde el inicio del estudio. Para el análisis estadístico de los datos se efectuó preliminarmente análisis univariado y luego análisis multivariado mediante regresión logística, utilizando las variables independientes propuestas. Fueron considerados estadísticamente significativos aquellos hallazgos asociados a valores $\mathrm{p}$ menores de 0,05 . Se calcularon valores de Odds Ratio (OR) crudos y ajustados con sus correspondientes intervalos de $95 \%$ de confianza ${ }^{16-19}$.

\section{RESULTADOS}

\section{a) Características de la población:}

La población encuestada estuvo compuesta por 1.208 sujetos con una mayor proporción de mujeres $(61,6 \%)$ que de hombres $(38,4 \%)$. Un $44,7 \%$ de sujetos pertenecían al estrato socioeconómico medio, un $40 \%$ al estrato bajo y un $15,1 \%$ al alto.
La Tabla 1 muestra el promedio de escolaridad por estrato socio-económico. Existen marcadas diferencias de escolaridad entre los tres niveles analizados. El promedio de edad fue mayor en los participantes del estrato alto, seguido del medio y luego del estrato bajo. La diferencia significativa está explicada fundamentalmente por los participantes del nivel socioeconómico alto que presenta una población con mayor promedio de edad.

\section{b) Número de sesiones necesarias para obte- ner una espirometría satisfactoria}

La espirometría se realizó en 1.168 sujetos debido a que 40 sujetos tenían criterios de exclusión espirométrica. La Figura 1 muestra la secuencia de repetición de las espirometrías en un diagrama de flujo. En 1.037 sujetos $(88,8 \%$ de la muestra) se obtuvo una espirometría satisfactoria en la primera entrevista mientras que 131 individuos $(11,2 \%)$ fueron incapaces de realizar una espirometría de calidad en esta primera sesión. De ellos, 89 aceptaron repetir la prueba en una segunda sesión de espirometría en terreno y 71 lograron una espirometría satisfactoria. Sólo 10 de los 18 participantes restantes aceptaron repetir la espirometría en una tercera oportunidad en la cual se obtuvo 7 espirometrías satisfactorias. Sólo dos de los 3 sujetos restantes aceptó repetir la espirometría por cuarta vez, la cual fue de calidad adecuada en sólo un sujeto. En resumen, en sólo 52 (4,45\%) de los 1.168 individuos no fue posible obtener una prueba satisfactoria. Es importante destacar que 42 de estos 52 sujetos no aceptaron realizar una segunda prueba espirométrica.

\section{c) Causas de espirometrías calificadas como insatisfactorias}

De las 131 espirometrías insatisfactorias, la mayoría presentó más de un criterio por el cual no aprobaron el control de calidad. Un $77,1 \%$ de las espirometrías no alcanzaron los criterios preestablecidos de aceptabilidad. Las causas más frecuentes fueron curvas con esfuerzo espira-

Tabla 1.Características de la población entrevistada

\begin{tabular}{llrrcc}
\hline Variables & & \multicolumn{3}{c}{ Nivel socioeconómico } \\
Medio & Blto & $\begin{array}{c}\text { Bajo } \\
\text { p }\end{array}$ \\
\hline Población & $(\%)$ & 15,1 & 44,7 & 40,1 & - \\
Escolaridad & (años) & $13,3 \pm 4,7$ & $9,1 \pm 4,3$ & $7,9 \pm 4,1$ & $<0,001$ \\
Edad & (años) & $60 \pm 12,8$ & $56 \pm 12,3$ & $55 \pm 11,1$ & $<0,001$ \\
\hline
\end{tabular}




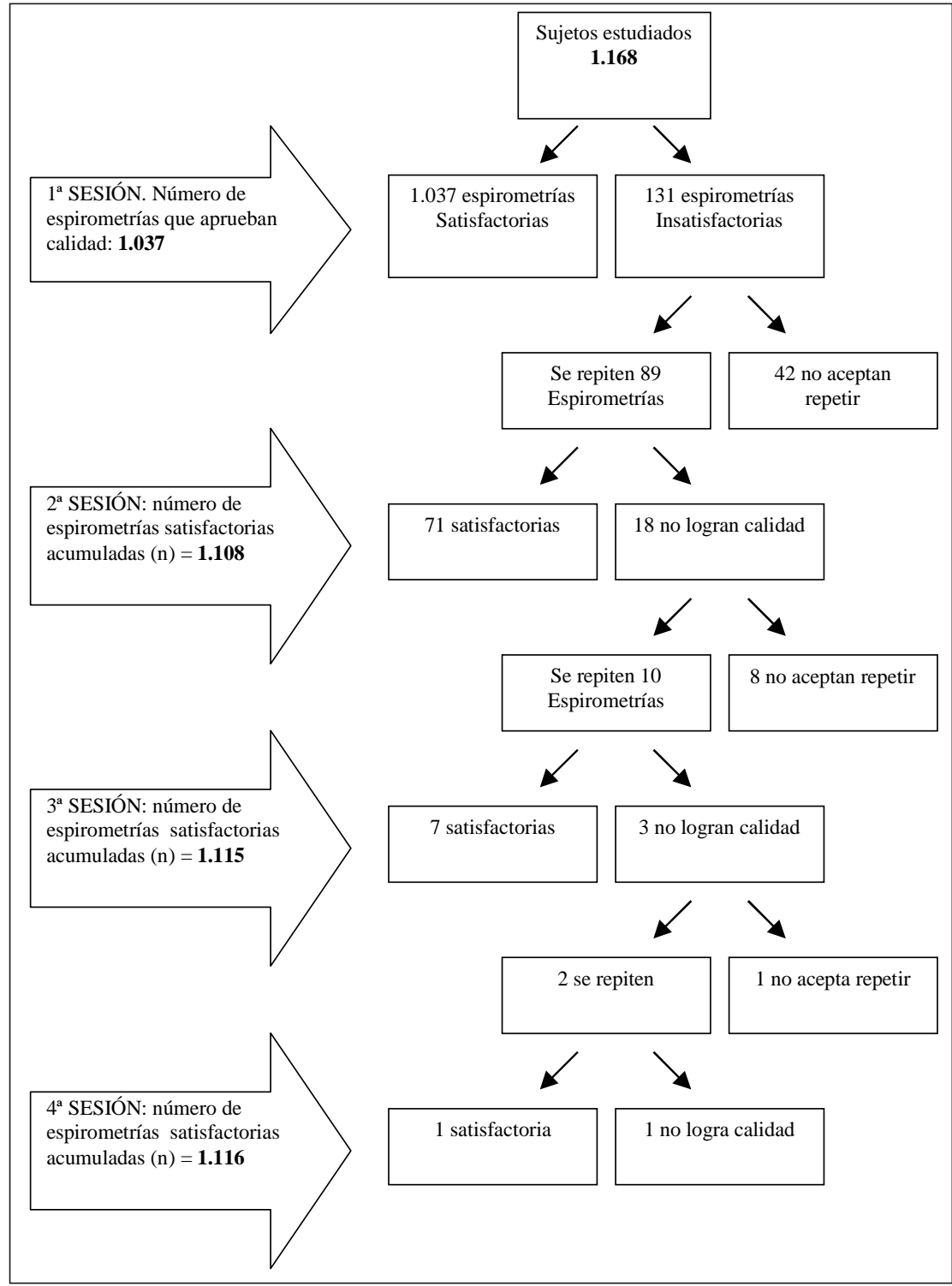

Figura 1. Diagrama de flujo que muestra la secuencia de repetición de las espirometrías hasta obtener una prueba satisfactoria.

torio variable, variaciones en el PEF mayores a un $10 \%$ y tiempo espiratorio inferior a $6 \mathrm{~s}$.

Un $67,9 \%$ de las espirometrías fueron insatisfactorias por falta de reproducibilidad, y un $36,6 \%$ de ellas presentaron caída en la CVF post broncodilatador sin disminución concomitante del $\mathrm{VEF}_{1}$.

\section{d) Calidad de las espirometrías a medida que transcurre el estudio}

La Figura 2 ilustra como a medida que el estudio progresó en el tiempo, se observó una tendencia a disminuir la tasa de fracasos en la primera sesión de espirometría. No obstante esta tendencia no fue estadísticamente significativa. e) Variables asociadas a la probabilidad de fracaso en obtener una espirometría técnicamente satisfactoria en la primera sesión

La Tabla 2 muestra los resultados del análisis univariado de las 10 variables explicatorias estudiadas. Se observa que sólo las variables fumador actual, edad y años de escolaridad se relacionaron significativamente con el fracaso de obtener una espirometría de calidad en la primera sesión.

El análisis multivariado de regresión logística mantuvo en el modelo sólo a las variables edad y años de instrucción como variables asociadas significativamente a la necesidad de repetir la espirometría (Tabla 3). 


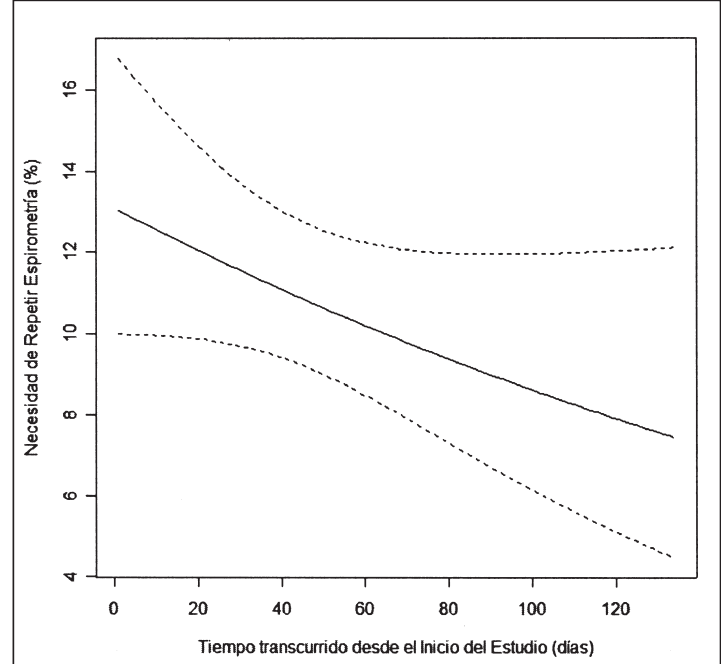

Figura 2. Tiempo transcurrido desde inicio del estudio y fracaso de la espirometría en la primera sesión.

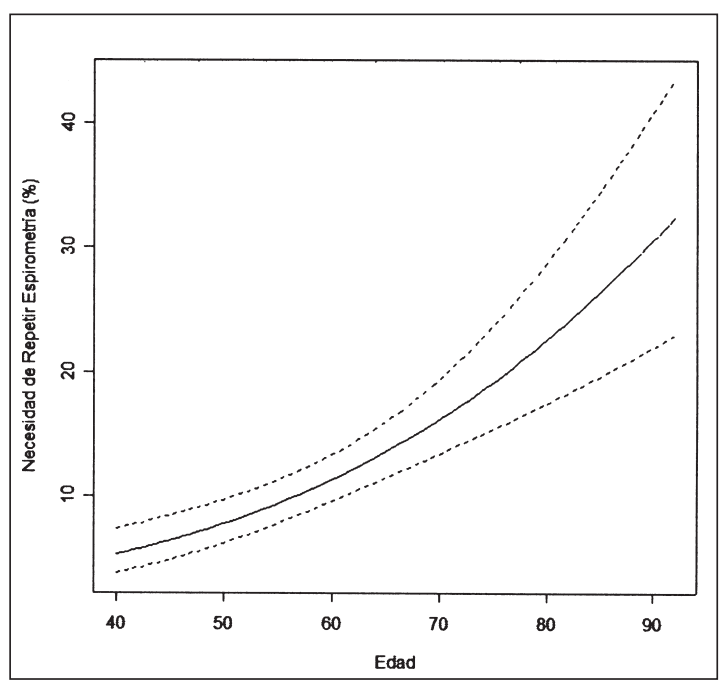

Figura 3. Fracaso de la espirometría en la primera sesión y edad del sujeto evaluado.

Tabla 2. Resultados del análisis univariado

\begin{tabular}{lccc}
\hline Variables & Grados deLibertad & Devianza & Valor $\mathbf{p}$ \\
\hline Tiempo transcurrido desde el inicio del estudio & 1 & 2,78 & 0,095 \\
Sexo & 1 & 0,69 & 0,406 \\
Nivel socioeconómico & 2 & 0,58 & 0,748 \\
Condición de Fumador & 1 & $\mathbf{7 , 7 3}$ & $\mathbf{0 , 0 0 5}$ \\
Edad & 1 & $\mathbf{3 1 , 9 4}$ & $<\mathbf{0 , 0 0 0 1}$ \\
Años de instrucción & 1 & $\mathbf{1 6 , 6 0}$ & $<\mathbf{0 , 0 0 0 1}$ \\
Antecedente de espirometría previa & 1 & 0,00 & 0,956 \\
Antecedente de resfrío & 1 & 2,29 & 0,130 \\
Índice de masa corporal & 1 & 0,75 & 0,386 \\
Técnico & 15 & 19,06 & 0,211 \\
\hline
\end{tabular}

Tabla 3. Resultados del análisis multivariado de regresión logística

\begin{tabular}{lcrc}
\hline Variables & $\begin{array}{c}\text { Grados de } \\
\text { Libertad }\end{array}$ & $\begin{array}{c}\text { Desviación } \\
\text { estándar }\end{array}$ & $\mathbf{p}$ \\
\hline Edad & 1 & 31,82 & $<0,0001$ \\
Años de educación & 1 & 5,22 & 0,022 \\
Fumador & 1 & 1,12 & 0,290 \\
\hline
\end{tabular}

La Figura 3 ilustra la asociación directa encontrada entre la edad del sujeto participante y la probabilidad de fracaso en la primera espirometría (OR 1,04 (95\% IC: 1,02-1,05).

Se observa además, que desde los 70 años se produce un incremento importante de la necesidad de repetir la prueba.

La Figura 4 ilustra la relación inversa encontrada entre la probabilidad de fracaso de la espirometría la primera vez que se realiza y los años de instrucción del sujeto (OR 0,94; 95\% IC: 0,90-0,98). 


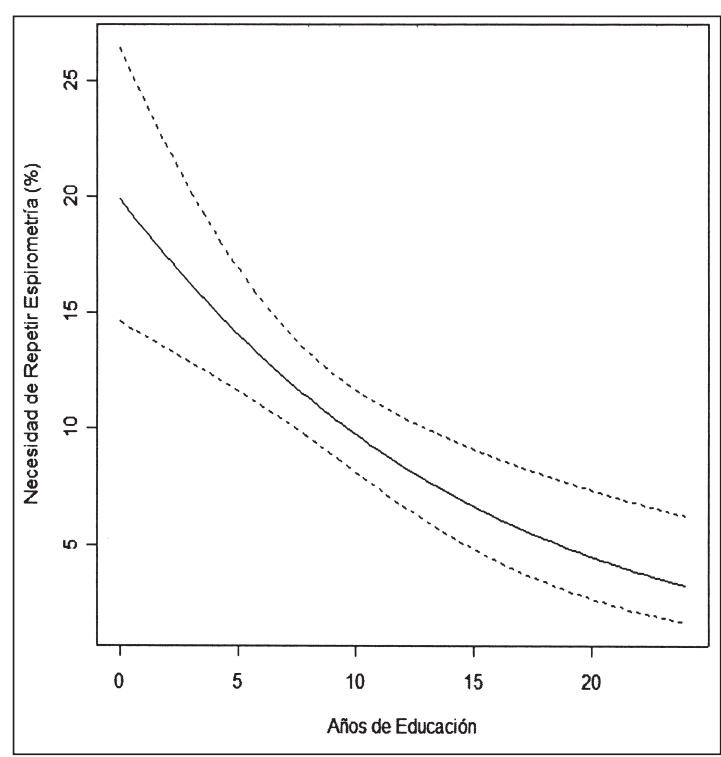

Figura 4. Años de instrucción del entrevistado y necesidad de repetición de espirometría.

\section{DISCUSIÓN}

Los principales resultados del presente estudio demuestran que, en un estudio epidemiológico de campo, empleando personal técnico no especializado en función pulmonar pero adecuadamente entrenado: 1) fue posible obtener espirometrías de buena calidad en la mayoría de los sujetos durante la primera entrevista; 2) las principales causas que determinaron la necesidad de repetir la espirometría fueron no cumplir los criterios técnicos de aceptabilidad y falta de reproducibilidad; 3) los factores significativamente relacionados con el fracaso espirométrico fueron la mayor edad y el bajo nivel de escolaridad del sujeto examinado y 4) el desempeño del técnico no fue un factor determinante de la mala calidad de la espirometría.

Diversos estudios en la literatura internacional atribuyen las causas de variabilidad de los resultados espirométricos a múltiples factores, tales como el personal técnico que realiza el examen, el tipo de espirómetro empleado, la calibración realizada y las características del sujeto estudiado $2,4,6,16,20,21$

Un $88,8 \%$ de la muestra estudiada en nuestro proyecto fue capaz de realizar una espirometría aceptable y reproducible en la primera sesión de estudio, porcentaje que aumenta a 95,5\% al incluir en el análisis las subsiguientes sesiones de repetición contemplados en el protocolo. Estos resultados concuerdan con los obtenidos en otros estudios realizados en laboratorios de función pulmonar con personal altamente especializa$\mathrm{do}^{2,6,16}$. Es así como la reproducibilidad comunicada por Enright y colaboradores ${ }^{16}$ en un estudio retrospectivo que analizó las espirometrías aceptables realizadas en un laboratorio especializado en 18.000 pacientes entre 20 y 90 años de edad fue también de $95 \%$. A diferencia de lo observado en nuestro estudio, la edad no constituyó un factor que influyera significativamente en la calidad de las espirometrías como tampoco el sexo, la presencia de enfermedad o su gravedad o el tabaquismo actual, factores que sólo determinaron entre un 2 y $4 \%$ de la variabilidad de la prueba, por lo que concluyeron que la habilidad para cumplir con las metas de reproducibilidad espirométrica depende fundamentalmente de la capacidad y perseverancia del personal técnico.

En este mismo sentido apuntan las conclusiones de Upton y colaboradores ${ }^{21}$ en un estudio epidemiológico sobre enfermedades cardiorrespiratorias con espirometrías realizadas en una clínica comunitaria. Ellos demostraron que la retroalimentación entregada al personal técnico fue un factor determinante que permitió mejorar en forma muy importante el nivel de aceptabilidad y de reproducibilidad de las espirometrías. Dicha realimentación se inició en la semana 15 del estudio y el personal técnico deficiente recibió entrenamiento adicional lo que determinó que las espirometrías realizadas en los últimos 6 meses del estudio fueran de calidad superior a la lograda en los tres primeros meses. En cambio, en el presente estudio las enfermeras recibieron retroalimentación individual, cada vez que la espirometría no alcanzaba la calidad requerida y también grupalmente en dos oportunidades con el propósito de reforzar la técnica empleada y mejorar la calidad. El análisis univariado no demostró que el personal técnico fuera un factor determinante de la necesidad de repetir la espirometría.

El impacto del personal técnico en la evaluación de la función pulmonar en estudios longitudinales, ha sido catalogado también como importante por Pérez Padilla y colaboradores ${ }^{4}$, quienes destacan que el entrenamiento y la permanente evaluación es esencial. En un estudio longitudinal realizado en niños ellos observaron que la calidad de la espirometría aumentó en la segunda fase del estudio, debido al efecto de aprendizaje tanto de los niños como de los técnicos y a una mejor interacción entre ellos.

De acuerdo a Enright y colaboradores ${ }^{16}$ el monitoreo frecuente del desempeño de los téc- 
nicos se asocia a mejoría y mantenimiento de la calidad de las espirometrías.

Por el contrario, Kunzli y asociados 22 en el estudio poblacional destinado a evaluar el efecto de la polución ambiental sobre las enfermedades pulmonares, no encontraron relación entre el desempeño de los técnicos y los errores en la espirometría.

El tipo de espirómetro utilizado ha sido también considerado como un factor involucrado en la mala calidad de la prueba. Sin embargo, desde que se dispone de equipos de medición de óptima calidad, que muestran gráficamente al operador las curvas flujo-volumen y volumentiempo de cada espiración forzada y que, proporcionan información sobre la calidad de la maniobra realizada, resulta poco probable que el espirómetro juegue un rol determinante en la mala calidad de la prueba. Nosotros empleamos espirómetros portátiles que miden flujo a través de un sensor ultrasónico ${ }^{12}$. La medición de flujo con este tipo de sensores es independiente de la composición, presión, temperatura y humedad del gas, lo que elimina los errores derivados de esas variables. Los espirómetros se calibraron diariamente, siguiendo normas preestable$\operatorname{cidas}^{5,22}$ y los equipos cuya calibración sobrepasó sostenidamente el rango aceptable fueron retirados del estudio, razón por la cual los espirómetros no fueron considerados en el análisis de las variables explicatorias de fracaso.

Diversas variables relacionadas con las características de los pacientes se consideran factores relacionados con la obtención de espirometrías no satisfactorias. En nuestro estudio la mayor edad y el menor número de años de educación se relacionaron significativamente con el fracaso de la espirometría en la entrevista inicial.

A su vez, en el trabajo de Upton y asocia$\operatorname{dos}^{21}$, el análisis univariado determinó que la condición de arrendatario, carencia de educación superior, enfermedad limitante e IMC bajo 20 $\mathrm{kg} / \mathrm{m}^{2}$ se relacionaban con falla en la aceptabilidad del $\mathrm{VEF}_{1}$ o de la CVF y que además, pertenecer al sexo femenino, tener edad avanzada, o asma se relacionaron con una mala reproducibilidad. En cambio, en una población de mayor edad, Pezzoli y colaboradores ${ }^{23}$ observaron que el mal desempeño espirométrico se relacionaba con la edad sólo si se asociaba con una alteración cognoscitiva o funcional. También Bellia y colaboradores $^{24}$ en población mayor de 65 años encontraron que el compromiso cognoscitivo, la baja capacidad para caminar y el nivel educacional bajo eran factores independientes de inacep- tabilidad. En cambio el sexo masculino y la mayor edad constituían riesgo de mala reproducibilidad. Los autores concluyen que la realización de la espirometría es dificultosa en ancianos, pero un buen control de calidad permite obtener información confiable en la mayoría de estos pacientes.

En resumen, en este estudio epidemiológico de terreno, el $88,8 \%$ de la población estudiada realizó una espirometría de buena calidad en una primera entrevista y este porcentaje aumentó a $95,5 \%$ en la repetición del examen. Si se excluye a los 42 sujetos que rechazaron efectuar la espirometría en una segunda ocasión, este porcentaje se eleva a 99\%. Las variables que influyeron en el fracaso de obtener un examen satisfactorio en el primer intento fueron la mayor edad de los sujetos y el menor nivel de escolaridad. Concluimos que personal técnico sin experiencia previa en realizar espirometrías, pero adecuadamente entrenado y evaluado, y con retroalimentación permanente es capaz de lograr espirometrías en terreno de tan alta calidad como la que se exige para los laboratorios de función pulmonar debidamente acreditados y que los factores relacionados con la probabilidad de fracaso en la obtención de una espirometría de calidad en la primera sesión fueron la mayor edad de los sujetos y la menor escolaridad de éstos.

\section{BIBLIOGRAFÍA}

1.- CRAPO R O. Spirometry: Quality control and reproducibility criteria. Am Rev Respir Dis 1991; 143: 1212-3.

2.- ENRIGHT P L, JOHNSON L R, CONNETT J E, VOELKER H, BUIST A S. Spirometry in the Lung Health Study. Methods and quality control. Am Rev Respir Dis 1991; 143: 1215-23.

3.- CRAPO R O. Pulmonary function testing. N Engl J Med 1994; 331: 25-30.

4.- PEREZ-PADILlA R, REGALADO- PINEDA J, MENDOZA L, ROJAS R, TORRES V, BORJAABURTO V, et al. Spirometric variability in a longitudinal study of school-age children. Chest 2003; 123: 1090-5.

5.- AMERICAN THORACIC SOCIETY. Standardization of spirometry. Am J RespCrit Care Med 1995; 152: 1107-36.

6.- HANKINSON J L, MOON BANG K. Acceptability and reproducibility criteria of the American Thoracic Society as observed in a sample of the general population Am Rev Respir Dis1991;143:516-21.

7.- MENEZES A M, VICTORA C G, PEREZ-PADILLA R. and the Platino team. The Platino project: methodology of a multicenter prevalence survey of chronic obstructive pulmonary disease in major Latin American cities. BMC Medical Research Methodology 2004; 4: 15 (http://www.biomedcentral.com/1471$2288 / 4 / 15)$. 
8.- FERRIS B G. Epidemiology Standardization Project (American Thoracic Society). Am Rev Respir Dis 1978; 118: 1-120.

9.- CONNETT J E, KUSEK J W, BAILEY W C, O'HARA P, WU M, for the Lung Health Study research Group. Design of the Lung Health Study: a randomized clinical trial of early intervention for chronic obstructive pulmonary disease. Control Clin Trials 1993; 14: 3-19.

10.- BURNEY P G, LUCZYNSKA CHINN S, JARVISA D. The European Community Respiratory Health Survey. Eur Resp J 1994; 7: 954-60.

11.- WARE J E, KOSINKI M, KELLER S D. How to score the SF12 physical and mental health summary scales. 2nd edition. Boston: Health Institute, New England Medical Center 1995.

12.- MORTIMER K M, FALLOT A, BALMES J R, TAGER B I. Evaluating the use of a portable spirometer in a study of pediatric asthma. CHEST 2003; 123: 1899-907.

13.- BLAND J M, ALTMAN D G. Statistical methods for assessing agreement between two methods of clinical measurement. Lancet 1986; 8: 307-10.

14.- LOHMAN I G, ROCHE A F, MARTORELL R (ed). Anthropometric Standardization Reference Manual. Champaing. Illinois, USA. Human Kinetics Books, 1988.

15.- HABICHT J P. Standardization of quantitative epidemiological methods in the field. Bol Oficina Sanit Panam 1974; 76: 375-84.

16.- HASTIE T J, TIBSHIRANI R J. Generalized additive models. Chapman and Hall. New York, 1990.

17.- HOSMER D, LEMESHOW S. Applied logistic regression models. $2^{\text {nd }}$ ed. John Wiley and Sons, New
York, USA 2000.

18.- ROSSENBERG P S, KATKI H, SWANSON C A, BROWN M, WACHOLDER S, HOOVER R N. Quantifying epidemiologic risk factors using nonparametric regression: model selection remains the greatest challenge. Statist Med 1999; 3369-81.

19.- WOOD S N. Modeling and smoothing parameter estimation with multiple quadratic penalties J R Statist Soc 2000; B 62: 413-28.

20.- ENRIGHT P, KENNETH C, BECK C, DUANE L, SHERRILL. Repeatability of Spirometry in 18.000 adult patients. Am J Respir Crit Care Med 2003; 169: 235-8.

21.- UPTON M N, FERRELL C, BIDWELL C, MCCONNACHIE A, GOODFELLOW J, DAVEY S G, et al. Improving the quality of spirometry in an epidemiological study: The Renfrew-Paisley (Midspan) family study. Public Health 2000; 114: 353-60.

22.- KUNZLI N, ACKERMANN-LIEBRICH U, KELLER R, PERRUCHOUD A P, SCHINDLER C.Variability of $\mathrm{FVC}$ and $\mathrm{FEV}_{1}$ due to technician, team, device and subject in an eight centre study: three quality control studies in SAPALDIA.Swiss study on air pollution and lung disease in adults. Eur Respir J 1995; 8: 371-6.

23.- PEZZOLI L, GIARDINI G, CONSONNI S, DALLERA I, BILOTTA C, FERRARIO G, et al. Quality control of spirometric performance in older people. Age Aging 2003; 32: 43-6.

24.- BELLIA V, PISTELLI R, CATALANO F, ANTONELLIINCALZI, GRASSI V, MELILLO G, et al. Quality control of spirometry in the elderly. The SARA. study. Salute Respiration nell 'Anziano Respiratory Health in the Elderly. Am J Respir Crit Care Med 2000; 161: 1094-100. 